\title{
A generalized DEMATEL theory with a shrinkage coefficient for an indirect relation matrix
}

\author{
Hsiang-Chuan Liu ${ }^{1}$, Ben-Chang Shia ${ }^{2}$, Yih-Chang $\mathrm{Ou}^{3, a}$ and Huan-Wen Su ${ }^{4}$ \\ ${ }^{1}$ Department of Biomedical Informatics, Asia University, Taichung 41354, Taiwan \\ ${ }^{2}$ Big Data Research Center \& School of Management School of Health Care Administration, Taipei Medical \\ University, Taipei 11031, Taiwan \\ ${ }^{3}$ Department of Finance, Ling Tung University, Taichung 40852, Taiwan \\ ${ }^{4}$ Graduate School of Business Administration, Fu Jen Catholic University, Xinzhuang Dist., New Taipei City 24205, \\ Taiwan
}

\begin{abstract}
In this paper, a novel decision-making trial and evaluation laboratory (DEMATEL) theory with a shrinkage coefficient of indirect relation matrix is proposed, and a useful validity index, called Liu's validity index, is also proposed for evaluating the performance of any DEMATEL model. If the shrinkage coefficient of an indirect relation matrix is equal to 1 , then this new theory is identical to the traditional theory; in other words, it is a generalization of the traditional theory. Furthermore, the indirect relation is always considerably greater than the direct one in traditional DEMATEL theory, which is unreasonable and unfair because it overemphasizes the influence of the indirect relation. We prove in this paper that if the shrinkage coefficient is equal to 0.5 , then the indirect relation is less than its direct relation. Because the shrinkage coefficient belongs to $[0.5,1]$, according to Liu's validity index, we can find a more appropriate shrinkage coefficient to obtain a more efficient DEMATEL method. Some crucial properties of this new theory are discussed, and a simple example is provided to illustrate the advantages of the proposed theory.
\end{abstract}

\section{Introduction}

A decision-making trial and evaluation laboratory (DEMATEL) was developed by Gabus and Fontela [1] and Fontela and Gabus [2] for resolving complex and difficult problems. To date, DEMATEL has been widely used as one of the most effective tools for solving cause-effect relationships among evaluation factors [3-10], but the indirect relation of a DEMATEL model is always markedly greater than its direct relation, which is unreasonable and unfair because it overemphasizes the influence of the indirect relation. In this paper, to overcome this drawback, we propose an external shrinkage coefficient for constructing a reduced indirect relation matrix, such that the new indirect relation matrix is less than its direct relation matrix, and for evaluating the performance of any DEMATEL model. We also propose a useful validity index, called Liu's validity index, for evaluating the performance of any DEMATEL model. Subsequently, a generalized DEMATEL is derived and shown to be more useful and effective than previous DEMATEL models.

\footnotetext{
${ }^{a}$ Corresponding author : ycou@teamail.ltu.edu.tw
} 


\section{Traditional DEMATEL model}

The procedure of the traditional DEMATEL method is briefly introduced as follows:

Step 1: Calculate the initial direct relation matrix $Q$

If $n$ factors are asked by $N$ experts to evaluate the degree of direct influence between two factors through pair-wise comparison, then the degree to which expert $e$ perceives factor $i$ as affecting factor $j$ is expressed as

$$
q_{i j}^{(e)}, e=1,2, . ., N \cdot q_{i j}^{(e)} \in\{0,1,2,3,4\}, i, j=1,2, \ldots, n
$$

For each expert $e$, an individual direct relation matrix is constructed as

$$
Q_{e}=\left[q_{i j}^{(e)}\right]_{n \times n}, e=1,2, . ., N, q_{i i}^{(e)}=0, i=1,2, \ldots, n
$$

and we can obtain their average direct relation matrix, which is called the initial direct relation matrix $Q$

$$
Q=\left[q_{i j}\right]_{n \times n}=\frac{1}{N} \sum_{e=1}^{N} Q_{e}, q_{i j}=\frac{1}{N} \sum_{e=1}^{N} q_{i j}^{(e)}, i, j=1,2, \ldots, n
$$

Step 2: Calculate the direct relation matrix $A$

$$
A=\left[a_{i j}\right]_{n \times n}=\lambda^{-1} Q, \lambda=\max _{1 \leq i, j \leq n}\left\{\sum_{j=1}^{n} q_{i j}, \sum_{i=1}^{n} q_{i j}\right\}
$$

where

$$
a_{i i}=0, i=1,2, \ldots, n, \quad 0 \leq a_{i j} \leq 1, i \neq j, i, j=1,2, \ldots, n
$$

and

$$
0 \leq \sum_{i=1}^{n} a_{i j}, \sum_{j=1}^{n} a_{i j} \leq 1, i, j=1,2, \ldots, n
$$

Step 3: Calculate the indirect relation matrix $B$ and the total relation matrix $T$ On the basis of Markov chain theory, we have

$$
\begin{gathered}
\lim _{k \rightarrow \infty} A^{k}=0_{n \times n} \\
B=\left[b_{i j}\right]_{n \times n}=\lim _{k \rightarrow \infty}\left[A^{2}+A^{3}+\ldots+A^{k}\right]=A^{2}(I-A)^{-1}, \\
T=\left[t_{i j}\right]_{n \times n}=A+B=\left[\left(a_{i j}+b_{i j}\right)\right]_{n \times n}
\end{gathered}
$$

Step 4: Calculate the relation degree and prominence degree of each factor

$$
r_{i}=\sum_{j=1}^{n} t_{i j}, \quad c_{i}=\sum_{k=1}^{n} t_{k i}, \quad i=1,2, \ldots, n
$$

Where $r_{i}$ indicates the total dispatch of both direct and indirect effects that factor $i$ has on the other factors, and $c_{i}$ indicates the total direct and indirect effects that factor $i$ has on the other factors. 
The relation degree of factor $i$ is expressed as

$$
x_{i}=r_{i}-c_{i}, \quad i=1,2, \ldots, n
$$

The prominence degree of factor $i$ is expressed as

$$
y_{i}=r_{i}+c_{i}, i=1,2, \ldots, n
$$

The relation-prominence matrix is expressed as

$$
\left(x_{i}, y_{i}\right)_{i=1}^{n}
$$

Step 5: Set the threshold value $(\alpha)$

To eliminate some minor effect elements in matrix $T$ to derive the impact-relations map, Ou Yang et al. [4] suggested the following threshold value:

$$
\alpha_{Y}=\frac{1}{n^{2}} \sum_{i=1}^{n} \sum_{j=1}^{n} t_{i j}
$$

Li and Tzeng [5] suggested a more informative threshold value, $\alpha_{M}$, on the basis of their maximum mean de-entropy algorithm.

Step 6: Build a cause-effect relationship diagram

If $t_{i j}>\alpha_{Y}$, or $t_{i j}>\alpha_{M}$, then factor $i$ is a net dispatch node of factor $j$, and factor $j$ is a net receive node of factor $i$, which is expressed as

$$
\left(x_{i}, y_{i}\right) \rightarrow\left(x_{j}, y_{j}\right), \text { or }\left(x_{i}, y_{i}\right) \leftarrow\left(x_{j}, y_{j}\right) .
$$

The graph of $\left(x_{i}, y_{i}\right)_{i=1}^{n}$, which includes the net direct edges, can present a cause-effect relationship diagram.

\section{Critical properties of the generalized DEMATEL model}

In this paper, we consider the critical properties of the traditional DEMATEL model to derive a more effective DEMATEL model, and then propose a generalized DEMATEL model. The critical properties of the generalized DEMATEL model are briefly described as follows.

Theorem 1. Critical properties of the generalized DEMATEL model

If

(a) $A=\left[a_{i j}\right]_{n \times n}=\left[a_{I J}^{(1)}\right]_{n \times n}, A^{2}=\left[a_{I J}^{(2)}\right]_{n \times n}=\left[\sum_{k=1}^{n} a_{i k} a_{k j}\right]_{n \times n}, A^{m+1}=\left[a_{I J}^{(m+1)}\right]_{n \times n}=\left[\sum_{k=1}^{n} a_{i k}^{(m)} a_{k j}\right]$

where

$$
\begin{gathered}
a_{i i}=0, i=1,2, \ldots, n, a_{i j} \geq 0, i, j=1,2, \ldots, n, \sum_{j=1}^{n} a_{i j} \leq 1, \sum_{i=1}^{n} a_{i j} \leq 1 \\
\text { (b) } \delta^{(1)}=\max _{1 \leq i, j \leq n}\left\{\sum_{j=1}^{n} a_{i j}, \sum_{i=1}^{n} a_{i j}\right\}=1, \quad \delta^{(m)}=\max _{1 \leq i, j \leq n}\left\{\sum_{j=1}^{n} a_{i j}^{(m)}, \sum_{i=1}^{n} a_{i j}^{(m)}\right\}, m \geq 2
\end{gathered}
$$




$$
\begin{gathered}
\text { (c) } B=\left[b_{i j}\right]_{n \times n}=A^{2}(I-A)^{-1}, \mu=\max _{1 \leq i, j \leq n}\left\{\sum_{j=1}^{n} b_{i j}, \sum_{i=1}^{n} b_{i j}\right\} \\
\text { (d) } B_{d}=\left[b_{i j}^{(d)}\right]_{n \times n}=d A^{2}(I-d A)^{-1}, d \in[0.5,1], \mu_{d}=\max _{1 \leq i, j \leq n}\left\{\sum_{j=1}^{n} b_{i j}^{(d)}, \sum_{i=1}^{n} b_{i j}^{(d)}\right\}
\end{gathered}
$$

then

$$
\begin{gathered}
\text { (i) } \delta^{(m+1)} \leq \delta^{(m)} \leq 1, m \geq 2 \\
\text { (ii) } \delta^{(2)}<1 \Rightarrow \delta^{(m+2)}<\delta^{(m)} \leq 1, m \in N \\
\text { (iii) } \mu=\lim _{m \rightarrow \infty} \sum_{s=2}^{m} \delta^{(m)}, \mu_{d}=\lim _{m \rightarrow \infty} \sum_{s=2}^{m} d^{s-1} \delta^{s} \\
\text { (iv) } d=0.5 \Rightarrow 0<\mu_{0.5} \leq 1 \\
\text { (v) } d=\frac{1}{1+\delta^{(2)}} \Rightarrow \mu_{0.5} \leq \mu_{d} \leq 1
\end{gathered}
$$

Proof

(i) $\sum_{j=1}^{n} a_{i j}^{(2)}=\sum_{j=1}^{n}\left(\sum_{k=1}^{n} a_{i k} a_{k j}\right)=\sum_{k=1}^{n}\left(\sum_{j=1}^{n} a_{k j}\right) a_{i k} \leq \sum_{k=1}^{n} a_{i k}=\sum_{j=1}^{n} a_{i j} \leq 1$

$$
\Rightarrow \sum_{j=1}^{n} a_{i j}^{(2)} \leq \sum_{j=1}^{n} a_{i j} \leq 1 \text {, in general, } \sum_{j=1}^{n} a_{i j}^{(m+1)} \leq \sum_{j=1}^{n} a_{i j}^{(m)} \leq 1
$$

Similarly, $\sum_{i=1}^{n} a_{i j}^{(m+1)} \leq \sum_{i=1}^{n} a_{i j}^{(m)} \leq 1$, we can obtain $\delta^{(m+1)} \leq \delta^{(m)} \leq 1, m \geq 2$.

(ii) $\begin{aligned} \sum_{j=1}^{n} a_{i j}^{(3)} & =\sum_{j=1}^{n}\left(\sum_{k=1}^{n} a_{i k} a_{k j}^{(2)}\right)=\sum_{k=1}^{n}\left(\sum_{j=1}^{n} a_{k j}^{(2)}\right) a_{i k}=\left(\sum_{j=1}^{n} a_{k j}^{(2)}\right) \sum_{k=1}^{n} a_{i k}>\sum_{j=1}^{n} a_{i j} \leq 1 \\ \sum_{j=1}^{n} a_{i j}^{(4)} & =\sum_{j=1}^{n}\left(\sum_{k=1}^{n} a_{i k}^{(2)} a_{k j}^{(2)}\right)=\sum_{k=1}^{n}\left(\sum_{j=1}^{n} a_{k j}^{(2)}\right) a_{i k}^{(2)}=\left(\sum_{j=1}^{n} a_{k j}^{(2)}\right) \sum_{k=1}^{n} a_{i k}^{(2)}>\sum_{k=1}^{n} a_{i k}^{(2)} \leq 1\end{aligned}$ In general, $\sum_{j=1}^{n} a_{i j}^{(m+2)}<\sum_{j=1}^{n} a_{i j}^{(m)} \leq 1$, we can obtain $\delta^{(m+2)}<\delta^{(m)} \leq 1, m \in N$.

(iii) $\mu=\max _{1 \leq i, j \leq n}\left\{\sum_{j=1}^{n} b_{i j}, \sum_{i=1}^{n} b_{i j}\right\}=\lim _{m \rightarrow \infty} \sum_{k=1}^{m} \max _{1 \leq i, j \leq n}\left\{\sum_{j=1}^{n} a_{i j}^{(s)}, \sum_{i=1}^{n} a_{i j}^{(s)}\right\}=\lim _{m \rightarrow \infty} \sum_{s=1}^{m} \delta^{(s)}$

Similarly, we have $\mu_{d}=\lim _{m \rightarrow \infty} \sum_{s=2}^{m} d^{s-1} \delta^{s}$.

(iv) We know that $\mu_{d}=\lim _{m \rightarrow \infty} \sum_{s=2}^{m} d^{s-1} \delta^{(s)} \leq \delta^{(2)} \lim _{m \rightarrow \infty} \sum_{s=2}^{m} d^{s-1}=\delta^{(2)} \frac{d}{1-d}$.

Because $0<\delta^{(2)} \leq 1$, if $\frac{d}{1-d} \leq 1$, then $\mu_{d}=\lim _{m \rightarrow \infty} \sum_{s=2}^{m} d^{s-1} \delta^{(s)} \leq \delta^{(2)} \frac{d}{1-d} \leq 1$;

$$
\text { that is, } d=\frac{1}{2} \Rightarrow 0<\mu_{d}=\mu_{0.5} \leq 1 \text {. }
$$


(v) Because $\mu_{d}=\lim _{m \rightarrow \infty} \sum_{s=2}^{m} d^{s-1} \delta^{(s)}$, is an increasing function of $d$ and $\delta^{(2)}$ and

$$
\begin{aligned}
& 0<\delta^{(2)} \leq 1, \frac{d}{1-d} \leq 1 \text {, then } \mu_{d}=\lim _{m \rightarrow \infty} \sum_{s=2}^{m} d^{s-1} \delta^{(s)} \leq \delta^{(2)} \frac{d}{1-d} \leq 1, \\
& \text { let } \delta^{(2)} \frac{d}{1-d}=1 \Rightarrow \frac{d}{1-d}=\frac{1}{\delta^{(2)}} \Rightarrow 0.5 \leq d=\frac{1}{1+\delta^{(2)}} \leq 1 \text {, then } \mu_{0.5} \leq \mu_{d} \leq 1 .
\end{aligned}
$$

\section{Method of the generalized DEMATEL model}

Because the indirect relation of a traditional DEMATEL model is always markedly greater than its direct relation, it overemphasizes the influence of the indirect relation. To overcome this drawback, given the aforementioned properties, an external shrinkage coefficient, $d$, is provided for constructing a reduced indirect relation matrix, and a useful validity index, called Liu's validity index, is also proposed for evaluating the performance of any DEMATEL model. Subsequently, a generalized DEMATEL model is derived and shown to be more useful and effective than previous DEMATEL models.

$$
\begin{gathered}
B_{d}=\left[b_{i j}^{(d)}\right]_{n \times n}=d A^{2}(I-d A)^{-1}, d \in\left[\frac{1}{2}, 1\right] \\
T_{d}=\left[t_{i j}^{(d)}\right]_{n \times n}=A+B_{d}=\left[\left(a_{i j}+b_{i j}^{(d)}\right)\right], d \in\left[\frac{1}{2}, 1\right]
\end{gathered}
$$

The relation-prominence of the DEMATEL $\left(A, B_{d}\right)$ is defined as

$$
\begin{gathered}
R\left(A, B_{d}\right)=\left(x_{i}^{(d)}, y_{i}^{(d)}\right)_{i=1}^{n}=\left(r_{i}^{(d)}-c_{i}^{(d)}, r_{i}^{(d)}+c_{i}^{(d)}\right)_{i=1}^{n} \\
\text { where } \quad r_{i}^{(d)}=\sum_{j=1}^{n} t_{i j}^{(d)}, c_{i}^{(d)}=\sum_{k=1}^{n} t_{i j}^{(d)}, \quad i=1,2, \ldots, n \\
\alpha_{Y}=\frac{1}{n^{2}} \sum_{i=1}^{n} \sum_{j=1}^{n} t_{i j}^{(d)}
\end{gathered}
$$

If $t_{i j}^{(d)}>\alpha_{Y}$, or $t_{i j}^{(d)}>\alpha_{M}$, then factor $i$ is a net dispatch node of factor $j$, and factor $j$ is a net receive node of factor $i$, which can be expressed as

$$
\left(x_{i}^{(d)}, y_{i}^{(d)}\right) \rightarrow\left(x_{j}^{(d)}, y_{j}^{(d)}\right)
$$

The graph of $\left(x_{i}, y_{i}\right)_{i=1}^{n}$, which includes the net direct edges, can present a cause-effect relationship diagram.

If $d=1$, then the new DEMATEL $\left(A, B_{d}\right)$ is simply the traditional DEMATEL $(A, B)$. 
If $d=0.5$, then $\max _{1 \leq i, j \leq n}\left\{\sum_{j=1}^{n} b_{i j}^{(d)}, \sum_{i=1}^{n} b_{i j}^{(d)},\right\} \leq 1$, and the new $\operatorname{DEMATEL}\left(A, B_{d}\right)$ is feasible because the influence of its indirect relation is no greater than the influence of its direct relation.

To evaluate the performance of any DEMATEL model, a useful validity index, called Liu's validity index, is proposed as follows:

$$
\begin{aligned}
& V_{L}\left(A, B_{d}\right)=1-\frac{1}{1+5 \sqrt{\sum_{i=1}^{n} \sqrt{\left(x_{i}^{(d)}-\bar{x}_{d}\right)^{2}+\left(y_{i}^{(d)}-\bar{y}_{d}\right)^{2}}}} \\
& \text { where } \bar{x}_{d}=\frac{1}{n} \sum_{j=1}^{n} x_{i}^{(d)}, \bar{y}_{d}=\frac{1}{n} \sum_{j=1}^{n} y_{i}^{(d)}
\end{aligned}
$$

Note that Liu's validity index can also be used for comparing the performance of any two DEMATEL models.

Example 1.

If a direct relation matrix of a DEMATEL model is obtained as

$$
A=\left[a_{i j}\right]_{4 \times 4}=\left[\begin{array}{cccc}
0 & 0.36 & 0.32 & 0.32 \\
0.32 & 0 & 0.34 & 0.30 \\
0.34 & 0.30 & 0 & 0.30 \\
0.28 & 0.28 & 0.30 & 0
\end{array}\right]
$$

then we can obtain the following indirect relation matrix:

$$
B=\left[b_{i j}\right]_{4 \times 4}=A^{2}(I-A)^{-1}=\left[\begin{array}{llll}
3.9448 & 3.8483 & 3.9290 & 3.7995 \\
3.7483 & 3.8237 & 3.7991 & 3.6907 \\
3.6807 & 3.6963 & 3.8253 & 3.6331 \\
3.4499 & 3.4478 & 3.4963 & 3.4508
\end{array}\right]
$$

where $\mu=\max _{1 \leq i, j \leq n}\left\{\sum_{j=1}^{n} b_{i j}, \sum_{i=1}^{n}\right\}=15.5215 \gg \max _{1 \leq i, j \leq n}\left\{\sum_{j=1}^{n} a_{i j}, \sum_{i=1}^{n} a_{i j},\right\}=1$.

This indicates that the DEMATEL model overemphasizes the influence of the indirect relation. Subsequently, we can obtain the total relation matrix, $T=\left[t_{i j}\right]_{4 \times 4}$, and the relation-prominence matrix, $R(A, B)$, respectively, as follows:

$$
\begin{gathered}
T=\left[t_{i j}\right]_{4 \times 4}=A+B=\left[\left(a_{i j}+b_{i j}\right)\right]_{4 \times 4}=\left[\begin{array}{llll}
3.9448 & 4.2083 & 4.2490 & 4.1195 \\
4.0683 & 3.8237 & 4.1391 & 3.9907 \\
4.0207 & 3.9963 & 3.8253 & 3.9331 \\
3.7299 & 3.7278 & 3.7963 & 3.4508
\end{array}\right] \\
R(A, B)=\left(x_{i}, y_{i}\right)_{i=1}^{n}=\left(\begin{array}{ll}
0.7579 & 32.2851 \\
0.2658 & 31.7780 \\
-0.2344 & 31.7850 \\
-0.7893 & 30.1989
\end{array}\right) \\
\alpha_{Y}=\frac{1}{4^{2}} \sum_{i=1}^{4} \sum_{j=1}^{4} t_{i j}=3.9390
\end{gathered}
$$




$$
T^{*}=\left[\begin{array}{llll}
3.9448^{*} & 4.2083^{*} & 4.2490^{*} & 4.1195^{*} \\
4.0683^{*} & 3.8237 & 4.1391^{*} & 3.9907^{*} \\
4.0207^{*} & 3.9963^{*} & 3.8253 & 3.9331 \\
3.7299 & 3.7278 & 3.7963 & 3.4508
\end{array}\right]
$$

$t_{i j}>\alpha_{Y}=3.9390 \Rightarrow x_{i} \rightarrow x_{j}$ indicates that factor $x_{i}$ is a net dispatch node of factor $x_{j}$.

The graph of $\left(x_{i}, y_{i}\right)_{i=1}^{n}$, which includes the net direct edges, can present a cause-effect relationship diagram.

$$
V_{L}(A, B)=V_{L}\left(A, B_{1}\right)=1-\frac{1}{1+5 \sqrt{\sum_{i=1}^{n} \sqrt{\left(x_{i}-\bar{x}\right)^{2}+\left(y_{i}-\bar{y}\right)^{2}}}}=0.9015
$$

If we consider a new DEMATEL model with a shrinkage coefficient of 0.5 , we can obtain the responding indirect relation matrix $B_{0.5}$ and its total relation matrix $T_{0.5}$, respectively, as follows:

$$
\begin{gathered}
B_{0.5}=0.5 A^{2}(I-0.5 A)^{-1}=\left[\begin{array}{llll}
0.2538 & 0.1993 & 0.2144 & 0.2047 \\
0.2012 & 0.2450 & 0.2002 & 0.2012 \\
0.1916 & 0.2020 & 0.2450 & 0.1971 \\
0.1882 & 0.1879 & 0.1872 & 0.2182
\end{array}\right], \\
\text { then } \mu_{0.5}=0.8722<\max _{1 \leq i, j \leq n}\left\{\sum_{j=1}^{n} a_{i j}, \sum_{i=1}^{n} a_{i j}\right\}=1 \leq \mu=15.5215
\end{gathered}
$$

Formula (29) shows that the indirect relation is already less than its direct relation; we can then obtain its total relation matrix and relation-prominence matrix, respectively, as follows:

$$
\begin{gathered}
T_{0.5}=A+B_{0.5}=\left[\begin{array}{llll}
0.2538 & 0.5593 & 0.5344 & 0.5247 \\
0.5212 & 0.2450 & 0.5402 & 0.5012 \\
0.5316 & 0.5020 & 0.2450 & 0.4971 \\
0.4682 & 0.4679 & 0.4872 & 0.2182
\end{array}\right] \\
\alpha_{Y}=\frac{1}{4^{2}} \sum_{i=1}^{4} \sum_{j=1}^{4} t_{i j}^{(0.5)}=0.4735 \\
T_{0.5}^{*}=\left[\begin{array}{llll}
0.2538 & 0.5593^{*} & 0.5344^{*} & 0.5247^{*} \\
0.5212^{*} & 0.2450 & 0.5402^{*} & 0.5012^{*} \\
0.5316^{*} & 0.5020^{*} & 0.2450 & 0.4971^{*} \\
0.4682^{*} & 0.4679^{*} & 0.4872^{*} & 0.2182
\end{array}\right] \\
R\left(A, B_{0.5}\right)=\left(x_{i}^{(0.5)}, y_{i}^{(0.5)}\right)_{i=1}^{n}=\left(\begin{array}{ll}
0.0973 & 3.6469 \\
0.0334 & 3.5718 \\
-0.0312 & 3.5824 \\
-0.0996 & 3.3526
\end{array}\right)
\end{gathered}
$$


If $t_{i j}^{(0.5)}>\alpha_{Y}=0.4735$, then $x_{i}^{(0.5)} \rightarrow x_{j}^{(0.5)}$ indicates that factor $i$ is a net dispatch node of factor $j$.

The graph of $\left(x_{i}^{(0.5)}, y_{i}^{(0.5)}\right)_{i=1}^{n}$, which includes the net direct edges, can present a cause-effect relationship diagram.

$$
V_{L}\left(A, B_{0.5}\right)=1-\frac{1}{1+5 \sqrt{\sum_{i=1}^{n} \sqrt{\left(x_{i}^{(0.5)}-\bar{x}_{0.5}\right)^{2}+\left(y_{i}^{(0.5)}-\bar{y}_{0.5}\right)^{2}}}}=0.7653
$$

Hence, DEMATEL $\left(A, B_{0.5}\right)$ is more effective than DEMATEL $(A, B)$. Furthermore, according to Liu's validity index, we can choose another shrinkage coefficient in $[0.5,1]$ to find the superior DEMATEL model.

\section{Conclusion}

In this paper, we show that the indirect relation is always considerably greater than the direct relation in traditional DEMATEL theory; consequently, it overemphasizes the influence of the indirect relation. Additionally, a novel DEMATEL theory with a shrinkage coefficient of an indirect relation matrix is proposed, and a useful validity index, called Liu's validity index, is also proposed for evaluating the performance of any DEMATEL model. We prove that for any DEMATEL model, if the shrinkage coefficient is equal to 0.5 , then the indirect relation is less than its direct relation, and because the shrinkage coefficient belongs to $[0.5,1]$, according to Liu's validity index, we can determine a more appropriate shrinkage coefficient to obtain a more effective DEMATEL method. Some crucial properties of this new theory are discussed, and a simple example is provided in this paper to illustrate the advantages of the proposed theory.

\section{References}

1. A. Gabus and E. Fontela, Perceptions of the World Problematique: Communication Procedure, Communicating with those Bearing Collective Responsibility (Switzerland Geneva, 1973)

2. E. Fontela and A. Gabus, The DEMATEL observer (Switzerland Geneva, 1976)

3. G.H. Tzeng, C.H. Chiang, and C.W. Li, Expert. Syst. Appl., 32, 4 (2007)

4. Y.P.O. Yang, H.M. Shieh, J.D. Leu, and G.H. Tzeng, Int. J. Oper. Res., 5, 3 (2008)

5. C.W. Li and G.H. Tzeng, Expert. Syst. Appl., 36, 6 (2009)

6. C.L. Lin and G.H. Tzeng, Expert. Syst. Appl., 36, 6 (2009)

7. J.I. Shieh, H.H. Wu, and K.K. Huang, Knowl-based. Syst., 23, 3 (2010)

8. D. Sumrit and P. Anuntavoranich, Int. T. J. Eng. Manage. App. Sci. Technol., 4, 2 (2013)

9. C.W. Wang, Y.H. Lin, C.L. Chung, and M.T. Lee, Expert. Syst. Appl., 39, 5 (2012)

10. E. Falatoonitoosi, Z. Leman, S. Sorooshian, and M. Salimi, Res. J. Appl. Sci. Eng. Technol., 5, 13 (2013) 